\title{
Principais causas de condenação de fígado bovino em estabelecimento sob Serviço de Inspeção Federal na Zona da Mata mineira
}

\author{
[Main causes of bovine liver condemnation in an abattoir under the Federal Inspection Services in the \\ Zona da Mata of the state of Minas Gerais] \\ S.P. Souza ${ }^{1}$, M.C.A. Klem ${ }^{1}$, K.P. Costa ${ }^{2}$, L.F. Silva ${ }^{3 *}$ \\ ${ }^{1}$ Faculdade Vértice - Matipó, MG \\ ${ }^{2}$ Médica veterinária autônoma - Muriaé, MG \\ ${ }^{3}$ Universidade Federal de Viçosa - UFV - Viçosa, MG
}

\section{RESUMO}

Neste trabalho, foi avaliada a prevalência das principais causas de condenação de fígado bovino em abatedouro frigorífico sob Serviço de Inspeção Federal, situado na Zona da Mata mineira. Para obtenção dos dados, foram consultadas fichas relativas ao movimento mensal de abate de bovinos e de causas de condenação, no período compreendido entre 2007 e 2013, no referido estabelecimento. Durante esse período, foram abatidos 150.752 bovinos, e a prevalência de condenação hepática entre os anos variou de $9,38 \%$ a $14,09 \%$. As principais patologias envolvidas foram abscesso, telangiectasia e fasciolose, responsáveis por $32,03 \%, 29,20 \%$ e $21,95 \%$ das condenações, respectivamente. Além disso, foi observada tendência de redução na frequência de condenação por abscesso $(3,26 \%)$ e telangiectasia $(0,68 \%)$ ao ano e, em contraposição, aumento por fasciolose de 3,40\% ao ano. Conclui-se que as patologias responsáveis pelas condenações de fígado bovino foram abscesso, telangiectasia e fasciolose e que a inspeção post mortem tem grande importância no monitoramento da saúde e da produção animal e na qualidade da carne a ser comercializada.

Palavras-chave: inspeção de carnes, saúde pública, miúdos, impacto econômico

\begin{abstract}
The aim of the present study was to evaluate the frequency of the main causes for condemnation of bovine liver in an abattoir/cold store under the Federal Inspection Services, located in the Zona da Mata of the state of Minas Gerais, between 2007 and 2013. Study data was collated from forms containing information about the number of cattle slaughtered per month and the causes for liver condemnation at the studied abattoir. During the period under study, 150,752 cattle were slaughtered, and the frequency of liver condemnation varied between $9.38 \%$ and $14.09 \%$ from year to year. The main pathologies that led to liver condemnation were abscesses, telangiectasis, and fasciolosis, which were responsible for $32.03 \%, 29.20 \%$, and $21.95 \%$ of the condemnations, respectively. The frequency of condemnation because of abscesses and telangiectasis showed a trend of decline, $3.26 \%$ and $0.68 \%$ per year, respectively. The frequency of condemnation because of fasciolosis, however, showed a trend of increase by $3.40 \%$ per year. This study shows the usefulness of post mortem inspection of animal health and production monitoring.
\end{abstract}

Key-words: economic impact; offal; meat inspection, public health

\section{INTRODUÇ̃̃O}

No mercado das exportações de carne bovina realizadas pelo Brasil, $14 \%$ do valor deste é atribuído ao comércio dos miúdos (Exportações..., 2016). Desse modo, além da

Recebido em 11 de outubro de 2016

Aceito em 3 de dezembro de 2016

*Autor para correspondência (corresponding author)

E-mail: leticiaafs@gmail.com carne in natura, os miúdos, obtidos durante o processo de abate, são reais fontes de nutrientes alimentares para a população mundial e acrescentam valor econômico significativo à produção dos abatedouros frigoríficos (Chiba, 2005). Segundo Kale et al. (2011), é provável que os subprodutos alimentares se tornem ainda 
mais relevantes no futuro. Esses fatos justificam a extrema importância do Serviço de Inspeção veterinário para a saúde pública. Este tem como objetivo retirar do mercado produtos com alterações patológicas devido a zoonoses e também aqueles com aspecto repugnante. Tal prática tem o objetivo de tornar seguro o consumo humano dos alimentos inspecionados (Herenda et al., 1994).

As condenações relatadas apresentam perdas econômicas diretas para a indústria (Souza et al., 2007; Kale et al., 2011) e indiretas para o produtor. Isso ocorre porque animais com órgãos comprometidos não terão o mesmo desempenho produtivo e econômico quando comparados aos animais hígidos (Pereira et al., 2006; Mas-Coma et al., 2009; Dutra et al., 2010; Sanchez-Vazquez e Lewis, 2013). Desse modo, considerando que os órgãos são subprodutos que agregam valor na renda dos estabelecimentos de abate, torna-se necessário minimizar os prejuízos decorrentes da condenação deles (Chiba, 2005; Kale et al., 2011).

O fígado é um órgão vital devido às suas funções desintoxicantes e hemostáticas; porém, em virtude disso, torna-se suscetível às lesões causadas por afecções sistêmicas, parasitárias e infecciosas (Castro e Moreira, 2010). Ainda, por ser considerado um miúdo comestível e de valor comercial, a sua condenação durante as linhas de inspeção acarreta grande perda econômica, além de indicar alto grau de patologias nos animais abatidos (Brasil, 1997; Castro e Moreira, 2010).

Neste trabalho, foi avaliada a prevalência das principais causas de condenação de fígado bovino em um abatedouro frigorífico sob Serviço de Inspeção Federal (SIF), situado na Zona da Mata mineira, no período compreendido entre janeiro de 2007 e dezembro de 2013. O conhecimento dessas informações permitirá a gerência do frigorífico sugerir aos produtores medidas de controle a serem tomadas com o intuito de promover a melhoria da sanidade do rebanho, evitando, assim, perdas econômicas para ambos.

\section{MATERIAL E MÉTODOS}

Para a realização deste estudo, contou-se com a colaboração de um abatedouro frigorífico sob SIF, situado na região da Zona da Mata mineira, o qual o aprovou e disponibilizou os dados utilizados. Trata-se de um estudo retrospectivo, realizado por um levantamento epidemiológico de dados no referido estabelecimento.

Foram utilizadas, como fonte de informação, fichas relativas ao movimento mensal de abate de bovinos, no período compreendido entre janeiro de 2007 e dezembro de 2013, em um abatedouro frigorífico localizado na Zona da Mata mineira, sob regime do SIF. Essas fichas forneceram os registros das atividades de abate realizadas durante a rotina de inspeção post mortem, proporcionando informações como data do abate, número de animais abatidos, parte animal, diagnóstico e destinação das peças. Foram considerados os dados relativos às doenças diagnosticadas no fígado na linha de inspeção "E", em exame post mortem, responsáveis por condenação.

Os animais foram abatidos e inspecionados seguindo as normas dispostas pelo Ministério da Agricultura, Pecuária e Abastecimento (Mapa) (Brasil, 1971; Brasil, 1997). Os fígados nos quais foram detectadas alterações ou lesões durante a sua linha de inspeção foram condenados pelo agente de inspeção e encaminhados à graxaria, para elaboração de subprodutos não comestíveis. Os achados foram anotados em papeleta específica de condenação do Departamento de Inspeção Final e, em seguida, transferidos para o sistema online de notificação do Mapa, o Sistema de Informações Gerenciais do Serviço de Inspeção Federal.

Todos os dados coletados foram processados em planilhas do Microsoft Excel, e para analisar a tendência da série histórica da frequência, foi realizada a análise de regressão linear, no programa Sigma Plot 13.0.

\section{RESULTADOS E DISCUSSÃO}

No período de janeiro 2007 a dezembro de 2013, foram abatidos 150.752 bovinos no referido abatedouro frigorífico. Desse total, foram condenados 16.832 fígados por apresentarem algum tipo de lesão ou alteração hepática, registrando-se uma prevalência total de $11,17 \%$ na região da Zona da Mata mineira (Tab. 1). Nessa Tab., constata-se, ainda, que a frequência de condenações hepáticas apresentou discreto aumento de $11,33 \%$ em 2007 para $11,69 \%$ em 2013. 
Os resultados observados podem ser considerados baixos em comparação aos obtidos por Vieira et al. (2011), que encontraram percentual de condenação de fígados bovinos variando de 40,2\% a 48,5\%, no município de Atílio Vivacqua, no sul do estado do Espírito Santo, em um abatedouro frigorífico sob Serviço de Inspeção Estadual (SIE), entre os anos de
2008 e 2010. Entretanto, mostraram-se equivalentes em relação aos relatados por Silva et al. (2013), com 12,36\% de condenações hepáticas em um abatedouro frigorífico sob SIF em Uberlândia - MG, no ano de 2011. Valores semelhantes também foram obtidos por Baptista (2008), com 12,7\% de condenação hepática para todo o estado do Espírito Santo.

Tabela 1. Número de bovinos abatidos e prevalência das condenações de fígados, em um abatedouro frigorífico da região da Zona da Mata mineira, nos anos de 2007 a 2013, pelo Serviço de Inspeção Federal

\begin{tabular}{lccc}
\hline Ano & Número de bovinos abatidos & \multicolumn{2}{c}{ Fígados condenados } \\
& & N & \% \\
\hline 2007 & 17.820 & 2.019 & 11,33 \\
2008 & 31.094 & 2.917 & 9,38 \\
2009 & 21.413 & 2.380 & 11,11 \\
2010 & 22.193 & 3.126 & 14,09 \\
2011 & 19.424 & 2.294 & 11,81 \\
2012 & 21.906 & 2.120 & 9,68 \\
2013 & 16.902 & 1.976 & 11,69 \\
\hline Total & 150.752 & 16.832 & 11,17 \\
\hline
\end{tabular}

Essas condenações representaram perda econômica considerável ao longo do período estudado. Levando-se em consideração o total de fígados condenados (Tab. 1) e o valor pelo qual o referido estabelecimento repassa esse miúdo ao comércio ( $\mathrm{R} \$ 6,50)$, foram perdidos $\mathrm{R} \$ 109.408,00$, o que daria, em média, $\mathrm{R} \$$ $15.629,71$ por ano. Já um estudo realizado por Fruet et al. (2013) em quatro matadouros sob Serviço de Inspeção Municipal (SIM) do município de Santa Maria - RS, constatou-se que o fígado obteve atuação de maior relevância nas perdas por condenação no período de outubro de 2010 a setembro de 2011, o que resultou em prejuízo de $\mathrm{R} \$ 50.943,27$ para os estabelecimentos pesquisados.

Portanto, tendo em vista que o fígado é um dos miúdos mais valorizados comercialmente, fica evidente a importância de se evitarem tais condenações, por meio de planos de ações, estimulando o produtor a realizar um manejo sanitário adequado do rebanho, baseado em medidas profiláticas, a fim de otimizar a cadeia produtiva da carne (Sanchez-Vazqueze Lewis, 2013; Silva et al., 2013).

Entre as causas de condenações hepáticas ilustradas na Fig. 1, o abscesso apresentou a maior prevalência $(3,56 \%)$, seguido da telangiectasia $(3,29 \%)$ e da fasciolose $(2,44 \%)$. Porém, também foram diagnosticadas, embora em menor proporção, condenações por cirrose $(0,27 \%)$, peri-hepatite $(0,23 \%)$, hidatidose $(0,14 \%)$ e outras causas $(1,22 \%)$, como contaminação e congestão, que não foram contabilizadas individualmente (Fig. 1).

Em concordância com os resultados obtidos no presente trabalho, Castro e Moreira (2010) também identificaram o abscesso como a principal causa de condenação hepática, com prevalência de $2,40 \%$ em um abatedouro frigorífico sob SIF, no período de janeiro de 2005 a dezembro de 2009, no Triângulo Mineiro, Minas Gerais. Entretanto, segundo Vieira et al. (2011), essa foi a quarta maior causa de condenação na região sul do Espírito Santo, com 2,26\%, de 2008 a 2010. 


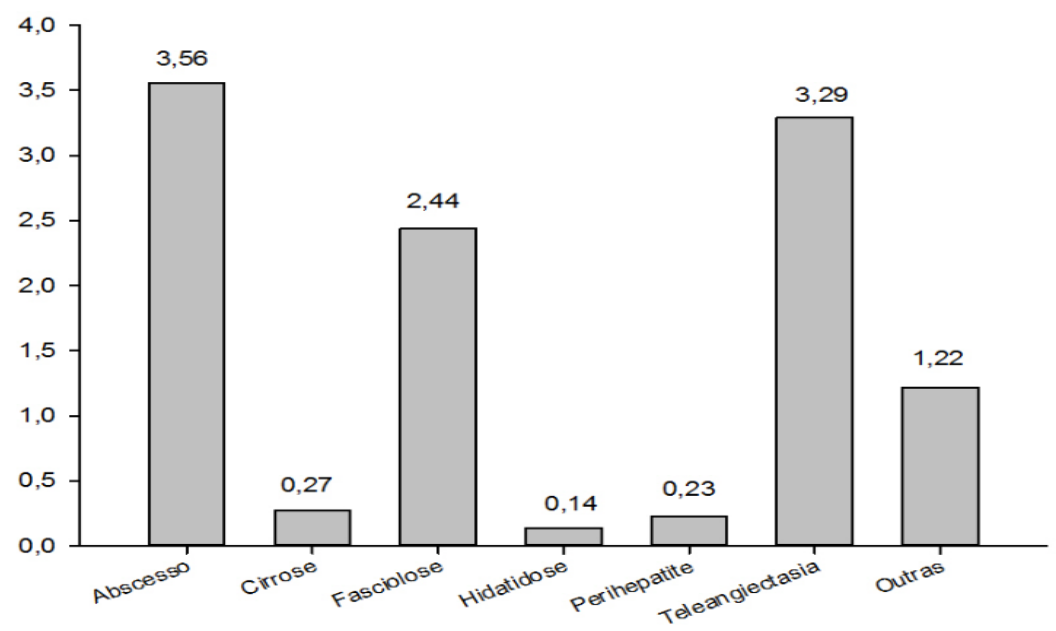

Figura 1. Prevalência (\%) das causas de condenações hepáticas diagnosticadas em um abatedouro frigorífico da Zona da Mata mineira, entre os anos de 2007 e 2013, pelo Serviço de Inspeção Federal.

Com relação às condenações por telangiectasia, Vieira et al. (2011) também a encontraram como a segunda principal causa, apesar de terem observado uma prevalência superior à deste estudo (5,30\%). Já segundo Castro e Moreira (2010), a telangiectasia foi a quarta maior causa de condenação hepática, mostrando-se com prevalência inferior, de apenas $0,59 \%$.

A prevalência encontrada para fasciolose neste trabalho foi consideravelmente superior à relatada por Aleixo et al. (2015), que encontraram uma prevalência de $0,01 \%$ em todo o estado de Minas Gerais; contudo, mostrou-se extremamente inferior à encontrada por Vieira et al. (2011), de 27,04\%, e por Gomes et al. (2002), que encontraram uma taxa de infecção de $15,38 \%$ em bovinos abatidos em Campos dos Goytacases - RJ. As grandes variações observadas quanto às condenações por fasciolose nos diferentes estados citados se dão, principalmente, em razão de o estado do Espírito Santo ser emergente para essa parasitose (Fraga, 2008).

Além disso, a ocorrência de fasciolose bovina é fortemente influenciada por uma associação de condições climáticas, como nível de precipitação e número de dias chuvosos, com alguns parâmetros ambientais, como o tipo de solo e a presença de áreas alagadiças, sendo esses considerados fatores de risco para a fasciolose e responsáveis pela distribuição espacial desigual da doença (Mas-Coma et al., 2009; Selemetas e Waal, 2015).
Em relação às demais doenças diagnosticadas (cirrose, peri-hepatite, hidatidose e outras), essas também se mostraram relevantes nos prejuízos por condenação, uma vez que, juntas, apresentam uma prevalência de 1,86\% (Fig. 1). Palma (2013) também encontrou prevalências semelhantes para cirrose $\quad(0,35 \%), \quad$ peri-hepatite $\quad(0,64 \%)$, hidatidose $(0,50 \%)$ e outras $(2,69 \%)$, as quais, somadas, apresentaram total de $4,18 \%$, o que mostra que, apesar de pouco prevalentes, quando somadas, acarretam grandes prejuízos ao abatedouro frigorífico, fazendo-se importante a prevenção de fatores que acarretam tais lesões.

Conforme mostra a Tab. 2, o percentual de condenações hepáticas por abscessos variou de $17,86 \%$ a $38,77 \%$ durante os sete anos estudados. Em concordância com os resultados deste trabalho, Castro e Moreira (2010) verificaram que os abscessos foram responsáveis por $37,27 \%$ das condenações hepáticas, na região do Triângulo Mineiro, entre janeiro de 2005 e dezembro de 2009.

Contudo, Silva et al. (2013) reportaram frequência superior a essas, também no estado de Minas Gerais, onde os abscessos foram responsáveis por $63,64 \%$ das condenações de fígados de bovinos abatidos em um abatedouro frigorífico sob SIF, no período de janeiro a dezembro de 2011, em Uberlândia. Já Vieira et al. (2011) demonstraram frequência inferior à deste trabalho, variando de $4,44 \%$ a $5,84 \%$ na região sul do Espírito Santo, no período de 2008 a 2010 . 
Tais achados podem ser explicados pelos diferentes manejos de criação adotados em cada região descrita, uma vez que maiores índices de abscessos hepáticos podem ser ocasionados devido ao sistema de confinamento (Vechiato et al., 2011).

A telangiectasia foi responsável por $18,15 \%$ a $48,83 \%$ das condenações hepáticas de 2007 a 2013 na região da Zona da Mata mineira (Tab. 2), aproximando-se tanto dos achados de Mendes e Pilati (2007), em cujo estudo 32,30\% dos fígados bovinos condenados em dois frigoríficos sob SIE, na região do Planalto Serrano e do Alto Vale do Itajaí, em Santa Catarina, foram em decorrência de telangiectasia, quanto dos achados de Silva et al. (2013), em que houve uma frequência de $19,18 \%$ de condenações em Uberlândia - MG, no ano de 2011.

Em concordância com os resultados apresentados, Pinto (2014) relata que, juntamente com os abscessos, a telangiectasia tem alcançado as mais altas frequências em fígado de bovinos abatidos, o que pode ser justificado, principalmente, pelo abate de animais mais velhos.

Ainda, pode ser observado, também na Tab. 2, que o percentual de condenação por fasciolose variou de $11,00 \%$ a $32,36 \%$ entre os anos estudados. Resultados semelhantes foram encontrados por Baptista (2008) e Mendes e Pilati (2007), em cujos estudos a fasciolose foi responsável por $38,60 \%$ das condenações hepáticas no Espírito Santo e por 18,50\% em Santa Catarina, respectivamente. Por outro lado, o percentual encontrado por Vieira et al. (2011) foi superior, variando de 58,51\% a $64,70 \%$.

Como dito anteriormente, essas variações nos achados de fasciolose podem estar relacionadas com o manejo dado em cada região, sendo o sistema extensivo o de maior risco para a manutenção do parasita (Andrews, 1999; Mendese Pilati, 2007). Além disso, podem ser influenciadas pela temperatura e umidade (climas temperados) e por condições ambientais (baixa altitude e hidrografia com presença de áreas alagadiças), já que esses fatores contribuem para a manutenção dos moluscos, hospedeiros intermediários, e, consequentemente, para a disseminação do agente etiológico (Roberts e Suhardono, 1996; Andrews, 1999).

Por fim, com base ainda nos dados fornecidos pela Tab. 2, foram realizadas análises de regressão linear das três principais causas de condenação hepática na Zona da Mata mineira e pôde-se observar que a frequência de condenações por abscesso e telangiectasia decresceram, enquanto a ocorrência de fasciolose aumentou ao longo dos sete anos pesquisados, como mostra a Fig. 2.

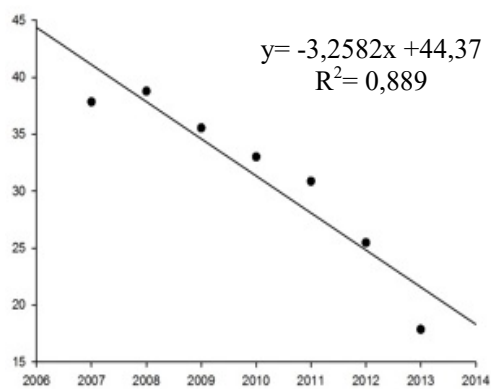

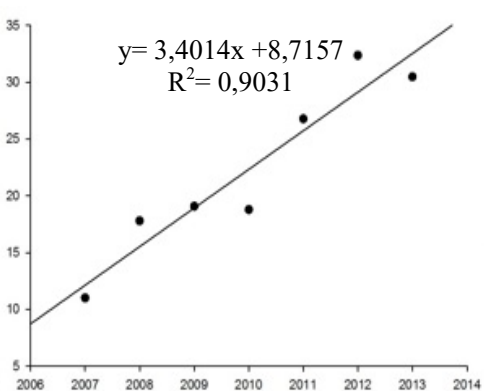

b

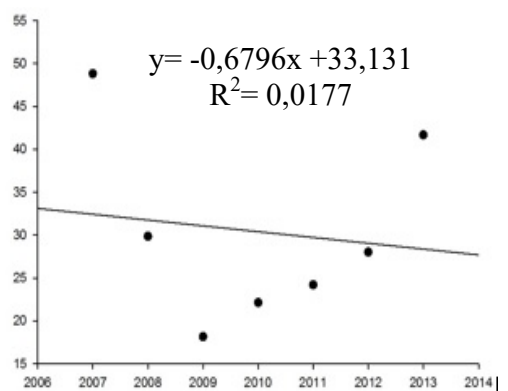

C

Figura 2. Análise de regressão linear da frequência (\%) das três principais causas de condenações de fígados bovinos, em um abatedouro frigorífico da Zona da Mata mineira, entre 2007 e 2013, pelo Serviço de Inspeção Federal: a) abscesso; b) fasciolose; c) telangiectasia. 
Principais causas de condenação...

Tabela 2. Quantitativo e percentual das causas de condenações de fígados bovinos na linha de inspeção "E", em um abatedouro frigorífico da Zona da Mata mineira, nos anos de 2007 a 2013, pelo Serviço de Inspeção Federal

\begin{tabular}{|c|c|c|c|c|c|c|c|c|c|c|c|c|c|c|}
\hline \multirow[t]{2}{*}{ Causa } & \multicolumn{2}{|c|}{2007} & \multicolumn{2}{|c|}{2008} & \multicolumn{2}{|c|}{2009} & \multicolumn{2}{|c|}{2010} & \multicolumn{2}{|c|}{2011} & \multicolumn{2}{|c|}{2012} & \multicolumn{2}{|c|}{2013} \\
\hline & $\mathrm{n}$ & $\%$ & $\mathrm{n}$ & $\%$ & $\mathrm{n}$ & $\%$ & $\mathrm{n}$ & $\%$ & $\mathrm{n}$ & $\%$ & $\mathrm{n}$ & $\%$ & $\mathrm{n}$ & $\%$ \\
\hline Cirrose & 8 & 0,40 & 4 & 0,14 & 124 & 5,21 & 173 & 5,53 & 62 & 2,70 & 34 & 1,60 & 0 & 0 \\
\hline Hidatidose & 9 & 0,45 & 3 & 0,10 & 62 & 2,61 & 65 & 2,08 & 35 & 1,53 & 36 & 1,70 & 8 & 0,40 \\
\hline Peri-hepatite & 0 & 0 & 0 & 0 & 116 & 4,87 & 144 & 4,61 & 33 & 1,44 & 46 & 2,17 & 14 & 0,71 \\
\hline Telangiectasia & 986 & 48,83 & 871 & 29,86 & 432 & 18,15 & 692 & 22,14 & 555 & 24,19 & 594 & 28,02 & 824 & 41,70 \\
\hline
\end{tabular}

Observa-se, na Fig. 2a, decréscimo no percentual de condenações por abscesso entre os anos de 2007 a 2013, com redução da frequência de $3,26 \%$ ao ano, com $89 \%$ de confiança. Trata-se de resultado positivo para a região da Zona da Mata mineira, pois as perdas decorrentes dos abscessos são as mais variadas. Dentre essas perdas, destacam-se a redução no ganho de peso dos animais, que pode chegar a $11 \%$, a eficiência alimentar desses animais, que sofre redução de até $9,7 \%$, e, também, a queda na taxa de crescimento de $5,8 \%$ a $12,7 \%$ naqueles animais que são acometidos, o que gera uma grande perda econômica para os pecuaristas e para os demais envolvidos nesse processo (Brink et al., 1990; Nagaraja e Chengappa, 1998; Nagaraja e Lechtenberg, 2007).

Já com relação ao percentual de condenação por fasciolose, foi observado um aumento de 3,40\% na frequência ao ano ( $90 \%$ de confiança), durante o período avaliado (Fig. 2b). Bernardo et al. (2011), ao avaliarem as condenações de fígados bovinos no sul do Espírito Santo, verificaram, para fasciolose, uma tendência histórica crescente entre os anos de 2006 a 2009, o que está de acordo com o descrito no presente trabalho. Esses resultados confirmam o descrito por Aleixo et al. (2015), que afirmaram que a fasciolose está, no momento, expandindo-se por todo o Brasil.

Trata-se de uma informação de grande importância, já que, além de aumentar as condenações hepáticas, a fasciolose também reflete negativamente no rendimento final da carcaça, uma vez que carcaças de bovinos parasitados por $F$. hepatica apresentam piores conformações, com menor espessura de gordura de cobertura e menor peso após a refrigeração, devido ao gotejamento excessivo (SanchezVazqueze Lewis, 2013).

Sobre o perfil de condenações por telangiectasia, devido às variações anuais no período em questão, a análise de regressão não foi confiável $(0,02 \%$ de confiança); no entanto, pode ser observada discreta tendência decrescente, com redução de $0,67 \%$ da frequência ao ano (Fig. 2c). Apesar de esse processo não apresentar reflexos sobre a saúde humana, esse comportamento decrescente é de grande importância econômica, já que os fígados afetados por essa patologia são rejeitados para consumo pelo aspecto repugnante que ela apresenta; além disso, o fato de a etiologia ser desconhecida dificulta a aplicação de métodos de prevenção (Brasil, 1997; Mendese Pilati, 2007).

\section{CONCLUSÕES}

As principais patologias responsáveis pelas condenações de fígados de bovinos na Zona da Mata mineira foram abscesso, telangiectasia e fasciolose. Essas condenações resultaram em perdas econômicas de significativa relevância tanto para o abatedouro frigorífico quanto para os produtores. A inspeção post mortem é uma ferramenta de grande importância para monitorar a saúde e a produção animal e para preservar a saúde pública. 


\section{REFERÊNCIAS}

ALEIXO, M.; FREITAS, D.F.; DUTRA, L.H. et al. Fasciola hepatica: epidemiology, perspectives in the diagnostic and the use of geoprocessing systems for prevalence studies. Semin. Ciênc. Agrar., v.36, p.1451-1466, 2015.

ANDREWS, S.J. The life cycle of Fasciola hepatica. In: DALTON, J. Fasciolosis. New York: CAB International, 1999. chapter 1, p.120.

BAPTISTA, A.T. Quantificações das condenações em vísceras de bovinos em 2007 nos matadouros-frigoríficos do estado do Espírito Santo registrados no Serviço de Inspeção Estadual. 2008. 22f. Monografia (PósGraduação em Higiene e Inspeção de Produtos de Origem Animal) - Universidade Castelo Branco, Vitória, ES.

BERNARDO, C.C.; CARNEIRO, M.B.; AVELAR, B.R. et al. Prevalência de condenação de fígados bovinos por fasciolose no sul do Espírito Santo: distribuição temporal e perdas econômicas. Rev. Bras. Parasitol. Vet., v.20, p.49-53, 2011.

BRASIL. Ministério da Agricultura, Pecuária e Abastecimento. Regulamento da Inspeção Industrial e Sanitária de Produtos de Origem Animal. Aprovado pelo Decreto $\mathrm{n}^{\circ} 30.691$, de 29-03-52, alterado pelos Decretos $\mathrm{n}^{\text {os }} 1.255$ de $25-06-62,1.236$ de $02-09-94, n^{0} 1.812$ de $08-02-$ 96 e n $^{\circ} 2.244$ de 04-06-97. Brasília, 1997. 174p.

BRASIL. Ministério da Agricultura, Pecuária e Abastecimento. Inspeção de carnes. Padronização de técnicas, instalações e equipamentos. Tomo I: Bovinos. Brasília: DIPOA/DICAR, 1971, 183p.

BRINK, D.R.; LOWRY, S.R.; STOCK, R.A.; PARROTT, J.C. Severity of liver abscess and efficiency IF feed utilization of feedlot cattle. $J$. Anim. Sci., v.68, p.1201-1207, 1990.

CASTRO, R.V.; MOREIRA, M.D. Ocorrências patológicas encontradas de rins e fígados bovinos em matadouro frigorífico do Triângulo Mineiro. $2010 . \quad$ Disponível em: $<$ http://www.fazu.br/ojs/index.php/posfazu/articl e/viewFile/343/249>. Acessado em: 26 set. 2015.

CHIBA, L.I. By-product feeds: animal origin. In: Encyclopedia of animal science. 2.ed. Alabama: Taylor e Francis, 2005. p.169-174.
DUTRA, L.H.; MOLENTO, M.B.; NAUMANN, C.R.C. et al. Mapping risk of bovine fasciolosis in the south of Brazil using geographic information systems. Vet. Parasitol., v.169, p.7681,2010

EXPORTAÇÕES brasileiras de carne bovina. São Paulo/Brasília: ABIEC, 2016. Disponível em $<$ http://www.abiec.com.br/download/Anual\%20j an\%20a\%20abr\%2016.pdf $>$. Acessado em: 10 jun. 2016.

FRAGA, J.C.L. Incidência da fasciolose hepática bovina no sul do Espírito Santo. 2008. 29f. Trabalho de conclusão de curso (Especialização) - Instituto Brasileiro de PósGraduação Qualittas, Universidade Castelo Branco, Rio de Janeiro, RJ.

FRUET, A.P.B.; FABRÍCIO, E.A.; KIRINUS, J.K. et al. Perdas econômicas oriundas das condenações de vísceras bovinas em matadouros de Santa Maria, Rio Grande do Sul. Rev. Bras. Cienc. Vet., v.20, p.99-103, 2013.

GOMES, F.F.; OLIVEIRA, F.C.R.; PILE, E.A.; LOPES, C.W.G. Estabelecimento de foco de fasciolose hepática em propriedade do município de Campos dos Goytacazes no estado do Rio de Janeiro, Brasil. Rev. Bras. Parasitol. Vet., v.22, p.53-56, 2002.

HERENDA， D.P.G.; CHAMBERS; A.; ETTRIQUI, P.; SENEVIRATNA, T.J.P.S. Manual on meat inspection for developing countries. Roma: Food and Agriculture Organization of the United Nations, 1994. p.234236.

KALE, M.C.; ARAL, Y.; AYDIN, E. et al. Determination of by-product economic values for slaughtered cattle and sheep. Kafkas Univ. Vet. Fak. Dergisi, v.17, p.551-556, 2011.

MAS-COMA, S.; VALERO, M.A.; BARGUES, M.D. Climate change effects on trematodiases, with emphasis on zoonotic fascioliasis and schistosomiasis. Vet. Parasitol., v.163, p.264280,2009 .

MENDES, R.E.; PILATI, C. Estudo morfológico de fígado de bovinos abatidos em frigorífico industriais sob inspeção estadual no Oeste e no Planalto de Santa Catarina, Brasil. Cienc. Rural, v.37, p.1728-1734, 2007. 
NAGARAJA, T.G.; CHENGAPPA, M.M. Liver abscess in feedlot: a review. J. Anim. Sci., v.76, p.287-298, 1998.

NAGARAJA， T.G.; LECHTENBERG， K.F. Liver abscess in feedlot cattle. Vet. Clin. N. Am. Food Anim. Pract., v.23, p.351-369, 2007.

PALMA, J.M. Principais lesões em carcaças e órgãos de bovinos oriundos de frigroríficos no Distrito Federal e Goiás. 2013. 28f. Monografia (Curso de Medicina Veterinária) - Faculdade de Agronomia e Medicina Veterinária, Universidade de Brasília, Brasília, DF.

PEREIRA, M.A.V.C.; SCHWANZ, V.S.; BARBOSA, C.G. Prevalência da cisticercose em carcaças de bovinos abatidos em matadourosfrigoríficos do estado do Rio de Janeiro, submetidos ao controle do serviço de inspeção federal (SIF- RJ), no período de 1997 a 2003. Arq. Inst. Biol., v.73, p.83-87, 2006.

PINTO, P.S.A. Inspeção e higiene de carnes. 2.ed. Viçosa, MG: UFV, 2014. 389p.

ROBERTS J.A.; SUHARDONO. Approaches to the control of fasciolosis in ruminants. Int. J. Parasitol., v.26, 971-981, 1996.

SANCHEZ-VAZQUEZ, M.J.; LEWIS, F.I. Investigating the impact of fasciolosis on cattle carcass performance. Vet. Parasitol., v.193, p.307-311, 2013.
SELEMETAS, N.; WAAL, T. Detection of major climatic and environmental predictors of livers fluke exposure risk in Ireland using spatial cluster analysis. Vet. Parasitol., v.209, p.242253, 2015.

SILVA, M.C.A.; MENDONÇA, G.A.; SOARES, D.B.; BUENO, J.P.R. Alterações anatomopatológicas identificadas na inspeção post mortem em bovinos no abatedouro frigorífico no município de Uberlândia- MG. Encicl. Biosfera, v.9, p.82-88, 2013.

SOUZA, V.K.; PESSÔA-SILVA, M.C.; KOWALCZUK, M. et al. Regiões anatômicas de maior ocorrência de Cysticercus bovis em bovinos submetidos à inspeção federal em matadouro-frigorífico no município de São José dos Pinhais, Paraná, de julho a dezembro de 2000. Rev. Bras. Parasitol. Vet., v.16, p.92-96, 2007.

VECHIATO, T.A.F.; MASCHIO, W.; BOM, L.C. et al. Estudo retrospectivo de abscessos hepáticos em bovinos abatidos em um frigorífico paulista. Braz. J. Vet. Res. Anim. Sci., v.48, p.384-391, 2011.

VIEIRA, N.P.; FARIA P.B.; MATTOS, M.R.; PEREIRA, A.A. Condenação de fígados bovinos na região sul do estado do Espírito Santo. Arq. Bras. Med. Vet. Zootec., v.63, p.1605-1608, 2011. 